Supporting Information

\title{
Novel cyclooctatetraene radical cation planarized by full annelation with bicyclo[2.1.1]hexene units
}

Tohru Nishinaga, Takayuki Uto and Koichi Komatsu* Institute for Chemical Research, Kyoto University, Uji, Kyoto 611-0011, Japan.

Cartesian coordinate of optimized structure of $1\left(D_{2 \mathrm{~d}}\right)$ at the B3LYP/6-31G(d) level

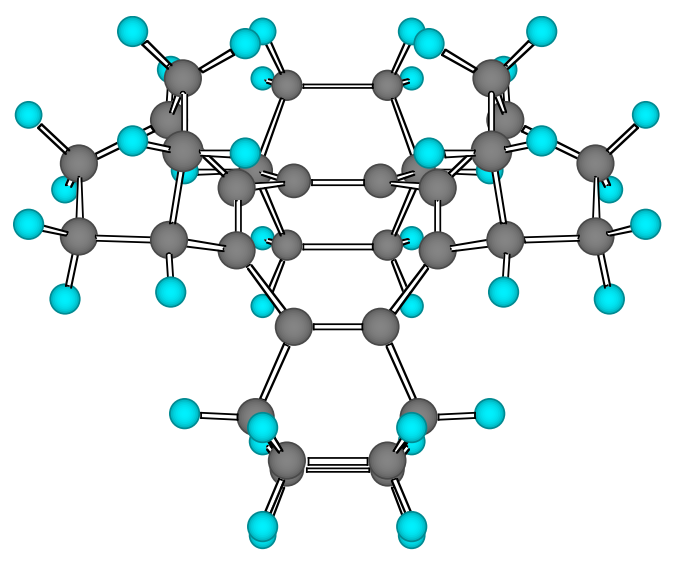
C -1.556203
0.676599
0.396813
C -1.556203
$-0.676599$
0.396813
C $-0.676599-1.556203$
$-0.396813$
C $0.676599-1.556203-0.396813$
C $1.556203 \quad-0.676599 \quad 0.396813$
C $1.556203 \quad 0.676599 \quad 0.396813$
C $0.676599 \quad 1.556203 \quad-0.396813$
C $-0.676599 \quad 1.556203-0.396813$
C $-2.634732 \quad 1.283060 \quad 1.287418$ 


$\begin{array}{lrrr}\text { C } & -2.634732 & -1.283060 & 1.287418 \\ \text { C } & -1.283060 & -2.634732 & -1.287418 \\ \text { C } & 1.283060 & -2.634732 & -1.287418 \\ \text { C } & 2.634732 & -1.283060 & 1.287418 \\ \text { C } & 2.634732 & 1.283060 & 1.287418 \\ \text { C } & 1.283060 & 2.634732 & -1.287418 \\ \text { C } & -1.283060 & 2.634732 & -1.287418 \\ \text { C } & -2.398235 & 0.777197 & 2.735531 \\ \text { C } & -4.026239 & 0.776267 & 0.823823 \\ \text { C } & -2.398235 & -0.777197 & 2.735531 \\ \text { C } & -4.026239 & -0.776267 & 0.823823 \\ \text { C } & -0.777197 & -2.398235 & -2.735531 \\ \text { C } & -0.776267 & -4.026239 & -0.823823 \\ \text { C } & 0.777197 & -2.398235 & -2.735531 \\ \text { C } & 0.776267 & -4.026239 & -0.823823 \\ \text { C } & 2.398235 & -0.777197 & 2.735531 \\ \text { C } & 4.026239 & -0.776267 & 0.823823 \\ \text { C } & 2.398235 & 0.777197 & 2.735531 \\ \text { C } & 4.026239 & 0.776267 & 0.823823 \\ \text { C } & 0.777197 & 2.398235 & -2.735531 \\ \text { C } & 0.776267 & 4.026239 & -0.823823 \\ \text { C } & -0.777197 & 2.398235 & -2.735531 \\ \text { C } & -0.776267 & 4.026239 & -0.823823 \\ \text { H } & -2.603719 & 2.377433 & 1.262543 \\ \text { H } & -2.603719 & -2.377433 & 1.262543 \\ \text { H } & -2.377433 & -2.603719 & -1.262543 \\ \text { H } & 2.377433 & -2.603719 & -1.262543 \\ \text { H } & 2.603719 & -2.377433 & 1.262543 \\ \text { H } & 2.603719 & 2.377433 & 1.262543 \\ \text { H } & 2.377433 & 2.603719 & -1.262543 \\ \text { H } & -2.377433 & 2.603719 & -1.262543 \\ \text { H } & -1.445855 & 1.168691 & 3.109500 \\ \text { H } & -3.185247 & 1.170318 & 3.391970 \\ \text { H } & -4.248301 & 1.167606 & -0.175207 \\ \text { H } & -4.798194 & 1.169433 & 1.497662\end{array}$




$\begin{array}{lrrr}\mathrm{H} & -1.445855 & -1.168691 & 3.109500 \\ \mathrm{H} & -3.185247 & -1.170318 & 3.391970 \\ \mathrm{H} & -4.248301 & -1.167606 & -0.175207 \\ \mathrm{H} & -4.798194 & -1.169433 & 1.497662 \\ \mathrm{H} & -1.168691 & -1.445855 & -3.109500 \\ \mathrm{H} & -1.170318 & -3.185247 & -3.391970 \\ \mathrm{H} & -1.167606 & -4.248301 & 0.175207 \\ \mathrm{H} & -1.169433 & -4.798194 & -1.497662 \\ \mathrm{H} & 1.168691 & -1.445855 & -3.109500 \\ \mathrm{H} & 1.170318 & -3.185247 & -3.391970 \\ \mathrm{H} & 1.167606 & -4.248301 & 0.175207 \\ \mathrm{H} & 1.169433 & -4.798194 & -1.497662 \\ \mathrm{H} & 1.445855 & -1.168691 & 3.109500 \\ \mathrm{H} & 3.185247 & -1.170318 & 3.391970 \\ \mathrm{H} & 4.248301 & -1.167606 & -0.175207 \\ \mathrm{H} & 4.798194 & -1.169433 & 1.497662 \\ \mathrm{H} & 1.445855 & 1.168691 & 3.109500 \\ \mathrm{H} & 3.185247 & 1.170318 & 3.391970 \\ \mathrm{H} & 4.248301 & 1.167606 & -0.175207 \\ \mathrm{H} & 4.798194 & 1.169433 & 1.497662 \\ \mathrm{H} & 1.168691 & 1.445855 & -3.109500 \\ \mathrm{H} & 1.170318 & 3.185247 & -3.391970 \\ \mathrm{H} & 1.167606 & 4.248301 & 0.175207 \\ \mathrm{H} & 1.169433 & 4.798194 & -1.497662 \\ \mathrm{H} & -1.168691 & 1.445855 & -3.109500 \\ \mathrm{H} & -1.170318 & 3.185247 & -3.391970 \\ \mathrm{H} & -1.167606 & 4.248301 & 0.175207 \\ \mathrm{H} & -1.169433 & 4.798194 & -1.497662 \\ & & & \\ \mathrm{HF} & -1243.4659411 \text { hartree } & \\ & & \end{array}$

Cartesian coordinate of optimized structure of $\mathbf{1}^{+}\left(D_{2 \mathrm{~d}}\right)$ at the B3LYP/6-31G(d) level 


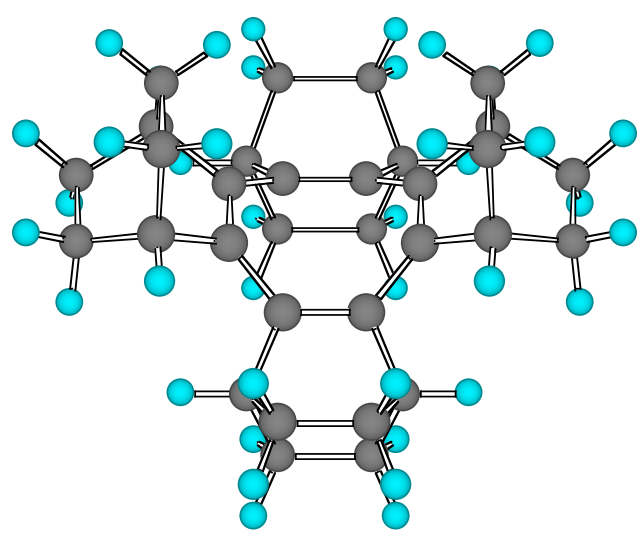
$\begin{array}{llll}\text { C } & 0.692954 & 1.571878 & 0.371147\end{array}$
$\begin{array}{llll}\text { C } & -0.692954 & 1.571878 & 0.371147\end{array}$
$\begin{array}{llll}\text { C } & -1.571878 & 0.692954 & -0.371147\end{array}$
$\begin{array}{llll}\text { C } & -1.571878 & -0.692954 & -0.371147\end{array}$
$\begin{array}{llll}\text { C } & -0.692954 & -1.571878 & 0.371147\end{array}$
$\begin{array}{llll}\text { C } & 0.692954 & -1.571878 & 0.371147\end{array}$
$\begin{array}{llll}\text { C } & 1.571878 & -0.692954 & -0.371147\end{array}$
$\begin{array}{llll}\text { C } & 1.571878 & 0.692954 & -0.371147\end{array}$
C $1.284938 \quad 2.757262 \quad 1.120110$
C $-1.284938 \quad 2.757262 \quad 1.120110$
C $-2.757262 \quad 1.284938-1.120110$
C $-2.757262-1.284938-1.120110$
$\begin{array}{llll}\text { C } & -1.284938 & -2.757262 & 1.120110\end{array}$
$\begin{array}{lrrr}\text { C } & 1.284938 & -2.757262 & 1.120110\end{array}$
C $2.757262 \quad-1.284938-1.120110$
C $2.757262 \quad 1.284938-1.120110$
$\begin{array}{llll}\text { C } & 0.775614 & 2.682457 & 2.583808\end{array}$
C $\quad 0.774530 \quad 4.081153 \quad 0.487531$
$\begin{array}{llll}\text { C } & -0.775614 & 2.682457 & 2.583808\end{array}$
$\begin{array}{llll}\text { C } & -0.774530 & 4.081153 & 0.487531\end{array}$
$\begin{array}{llll}\text { C } & -2.682457 & 0.775614 & -2.583808\end{array}$
$\begin{array}{llll}\text { C } & -4.081153 & 0.774530 & -0.487531\end{array}$
$\begin{array}{llll}\text { C } & -2.682457 & -0.775614 & -2.583808\end{array}$
$\begin{array}{llll}\text { C } & -4.081153 & -0.774530 & -0.487531\end{array}$
$\begin{array}{llll}\text { C } & -0.775614 & -2.682457 & 2.583808\end{array}$
C $-0.774530 \quad-4.081153 \quad 0.487531$ 


\begin{tabular}{|c|c|c|c|}
\hline C & 0.775614 & -2.682457 & 2.583808 \\
\hline C & 0.774530 & -4.081153 & 0.487531 \\
\hline C & 2.682457 & -0.775614 & -2.583808 \\
\hline C & 4.081153 & -0.774530 & -0.487531 \\
\hline$C$ & 2.682457 & 0.775614 & -2.583808 \\
\hline C & 4.081153 & 0.774530 & -0.487531 \\
\hline $\mathrm{H}$ & 2.376702 & 2.727250 & 1.105620 \\
\hline $\mathrm{H}$ & -2.376702 & 2.727250 & 1.105620 \\
\hline $\mathrm{H}$ & -2.727250 & 2.376702 & -1.105620 \\
\hline $\mathrm{H}$ & -2.727250 & -2.376702 & -1.105620 \\
\hline $\mathrm{H}$ & -2.376702 & -2.727250 & 1.105620 \\
\hline $\mathrm{H}$ & 2.376702 & -2.727250 & 1.105620 \\
\hline $\mathrm{H}$ & 2.727250 & -2.376702 & -1.105620 \\
\hline$H$ & 2.727250 & 2.376702 & -1.105620 \\
\hline $\mathrm{H}$ & 1.170655 & 1.784629 & 3.070353 \\
\hline $\mathrm{H}$ & 1.173597 & 3.541893 & 3.134229 \\
\hline $\mathrm{H}$ & 1.168664 & 4.188392 & -0.528523 \\
\hline $\mathrm{H}$ & 1.171154 & 4.919533 & 1.070524 \\
\hline $\mathrm{H}$ & -1.170655 & 1.784629 & 3.070353 \\
\hline $\mathrm{H}$ & -1.173597 & 3.541893 & 3.134229 \\
\hline $\mathrm{H}$ & -1.168664 & 4.188392 & -0.528523 \\
\hline $\mathrm{H}$ & -1.171154 & 4.919533 & 1.070524 \\
\hline $\mathrm{H}$ & -1.784629 & 1.170655 & -3.070353 \\
\hline $\mathrm{H}$ & -3.541893 & 1.173597 & -3.134229 \\
\hline $\mathrm{H}$ & -4.188392 & 1.168664 & 0.528523 \\
\hline $\mathrm{H}$ & -4.919533 & 1.171154 & -1.070524 \\
\hline $\mathrm{H}$ & -1.784629 & -1.170655 & -3.070353 \\
\hline $\mathrm{H}$ & -3.541893 & -1.173597 & -3.134229 \\
\hline $\mathrm{H}$ & -4.188392 & -1.168664 & 0.528523 \\
\hline $\mathrm{H}$ & -4.919533 & -1.171154 & -1.070524 \\
\hline $\mathrm{H}$ & -1.170655 & -1.784629 & 3.070353 \\
\hline $\mathrm{H}$ & -1.173597 & -3.541893 & 3.134229 \\
\hline $\mathrm{H}$ & -1.168664 & -4.188392 & -0.528523 \\
\hline $\mathrm{H}$ & -1.171154 & -4.919533 & 1.070524 \\
\hline $\mathrm{H}$ & 1.170655 & -1.784629 & 3.070353 \\
\hline
\end{tabular}




$\begin{array}{cccc}\text { H } & 1.173597 & -3.541893 & 3.134229 \\ \text { H } & 1.168664 & -4.188392 & -0.528523 \\ \text { H } & 1.171154 & -4.919533 & 1.070524 \\ \text { H } & 1.784629 & -1.170655 & -3.070353 \\ \text { H } & 3.541893 & -1.173597 & -3.134229 \\ \text { H } & 4.188392 & -1.168664 & 0.528523 \\ \text { H } & 4.919533 & -1.171154 & -1.070524 \\ \text { H } & 1.784629 & 1.170655 & -3.070353 \\ \text { H } & 3.541893 & 1.173597 & -3.134229 \\ \text { H } & 4.188392 & 1.168664 & 0.528523 \\ \text { H } & 4.919533 & 1.171154 & -1.070524\end{array}$

$H F=-1243.2469327$ hartree

Cartesian coordinate of optimized structure of $2\left(D_{4 \mathrm{~h}}\right)$ at the B3LYP/6-31G(d) level

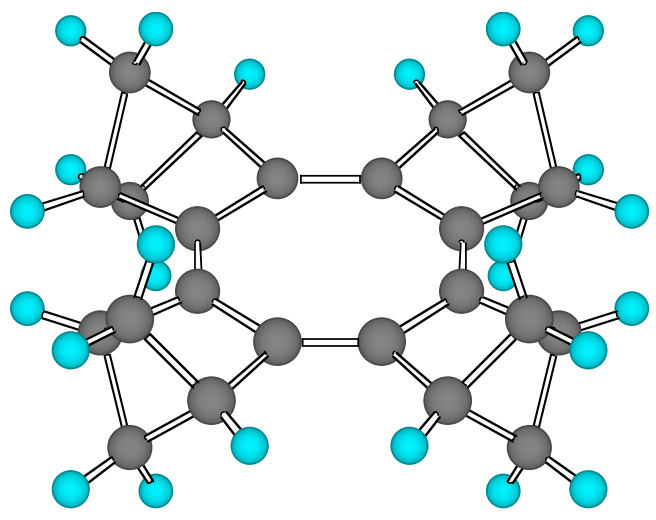
$\begin{array}{llll}\text { C } & 0.670964 & 1.733977 & 0.000000\end{array}$
$\begin{array}{llll}\text { C } & 1.733977 & 0.670964 & 0.000000\end{array}$
$\begin{array}{llll}\text { C } & 1.733977 & -0.670964 & 0.000000\end{array}$
$\begin{array}{llll}\text { C } & 0.670964 & -1.733977 & 0.000000\end{array}$
$\begin{array}{llll}\text { C } & -0.670964 & -1.733977 & 0.000000\end{array}$
$\begin{array}{llll}\text { C } & -1.733977 & -0.670964 & 0.000000\end{array}$
$\begin{array}{llll}\text { C } & -1.733977 & 0.670964 & 0.000000\end{array}$
$\begin{array}{llll}\text { C } & -0.670964 & 1.733977 & 0.000000\end{array}$
$\begin{array}{llll}\text { C } & 1.540397 & 2.989217 & 0.000000\end{array}$ 


$\begin{array}{rrrr}\text { C } & 2.989217 & 1.540397 & 0.000000 \\ \text { C } & 2.989217 & -1.540397 & 0.000000 \\ \text { C } & 1.540397 & -2.989217 & 0.000000 \\ \text { C } & -1.540397 & -2.989217 & 0.000000 \\ \text { C } & -2.989217 & -1.540397 & 0.000000 \\ \text { C } & -2.989217 & 1.540397 & 0.000000 \\ \text { C } & -1.540397 & 2.989217 & 0.000000 \\ \text { C } & 2.621020 & 2.621020 & 1.063434 \\ \text { C } & 2.621020 & 2.621020 & -1.063434 \\ \text { C } & 2.621020 & -2.621020 & 1.063434 \\ \text { C } & 2.621020 & -2.621020 & -1.063434 \\ \text { C } & -2.621020 & -2.621020 & 1.063434 \\ \text { C } & -2.621020 & -2.621020 & -1.063434 \\ \text { C } & -2.621020 & 2.621020 & 1.063434 \\ \text { C } & -2.621020 & 2.621020 & -1.063434 \\ \text { H } & 1.043412 & 3.958249 & 0.000000 \\ \text { H } & 3.958249 & 1.043412 & 0.000000 \\ \text { H } & 3.958249 & -1.043412 & 0.000000 \\ \text { H } & 1.043412 & -3.958249 & 0.000000 \\ \text { H } & -1.043412 & -3.958249 & 0.000000 \\ \text { H } & -3.958249 & -1.043412 & 0.000000 \\ \text { H } & -3.958249 & 1.043412 & 0.000000 \\ \text { H } & -1.043412 & 3.958249 & 0.000000 \\ \text { H } & 2.282854 & 2.282854 & 2.048015 \\ \text { H } & 3.392289 & 3.392289 & 1.162429 \\ \text { H } & 2.282854 & 2.282854 & -2.048015 \\ \text { H } & 3.392289 & 3.392289 & -1.162429 \\ \text { H } & 2.282854 & -2.282854 & 2.048015 \\ \text { H } & 3.392289 & -3.392289 & 1.162429 \\ \text { H } & 2.282854 & -2.282854 & -2.048015 \\ \text { H } & 3.392289 & -3.392289 & -1.162429 \\ \text { H } & -2.282854 & -2.282854 & 2.048015 \\ \text { H } & -3.392289 & -3.392289 & 1.162429 \\ \text { H } & -2.282854 & -2.282854 & -2.048015 \\ \text { H } & -3.392289 & -3.392289 & -1.162429\end{array}$




$$
\begin{array}{rrrr}
H & -2.282854 & 2.282854 & 2.048015 \\
H & -3.392289 & 3.392289 & 1.162429 \\
H & -2.282854 & 2.282854 & -2.048015 \\
H & -3.392289 & 3.392289 & -1.162429
\end{array}
$$

$H F=-928.7708315$ hartree

Cartesian coordinate of optimized structure of $2\left(D_{2 \mathrm{~d}}\right)$ at the B3LYP/6-31G(d) level

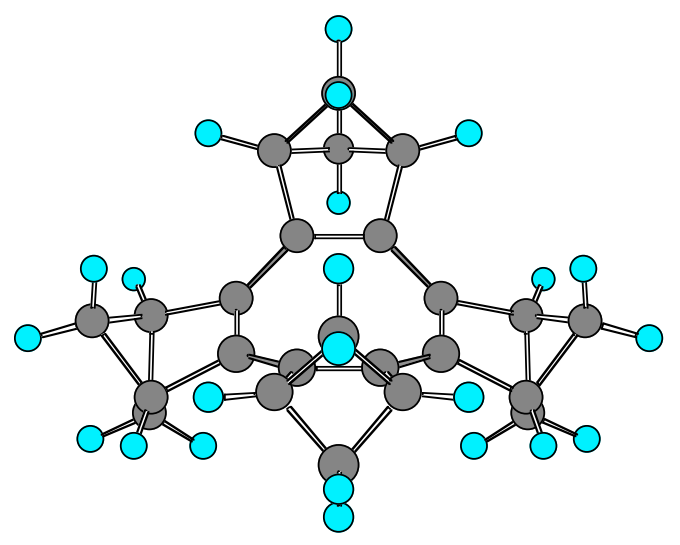
$\begin{array}{llll}\text { C } & 1.019392 & -3.009187 & 0.883514\end{array}$
C $0.678789-1.6453390 .249292$
$\begin{array}{llll}\text { C } & -0.678789 & -1.645339 & 0.249292\end{array}$
C $1.645339-0.678789-0.249292$
$\begin{array}{llll}\text { C } & -1.019392 & -3.009187 & 0.883514\end{array}$
C $-1.6453390 .678789-0.249292$
$\begin{array}{llll}\text { C } & 0.678789 & 1.645339 & 0.249292\end{array}$
C $3.009187-1.019392-0.883514$
C $1.6453390 .678789-0.249292$
C $3.009187 \quad 1.019392-0.883514$
C $-1.645339-0.678789-0.249292$
C $1.019392 \quad 3.009187 \quad 0.883514$
$\begin{array}{llll}\text { C } & -3.009187 & 1.019392 & -0.883514\end{array}$
C $-1.019392 \quad 3.009187 \quad 0.883514$
$\begin{array}{llll}\text { C } & -0.678789 & 1.645339 & 0.249292\end{array}$
$\begin{array}{llll}\text { C } & -3.009187 & -1.019392 & -0.883514\end{array}$ 


\begin{tabular}{|c|c|c|c|}
\hline & 171 & & \\
\hline & 0 & & \\
\hline & 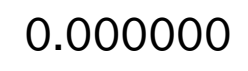 & & \\
\hline & 0.000000 & 031712 & 20704 \\
\hline & 3.031712 & & \\
\hline & 9356 & 0000 & \\
\hline & 0.000000 & -3.939356 & \\
\hline & 0.000000 & -3.031 & 2. \\
\hline & -2.071759 & -3.2 & 1. \\
\hline & 61 & & \\
\hline & 4.97 & 0.0 & -0.5 \\
\hline & -4.972200 & 0.000000 & \\
\hline & 0.0 & 4.9 & \\
\hline & $0.0 c$ & 5 & \\
\hline & 3.91 & & \\
\hline & 0.000000 & -3.9 & \\
\hline & 0.0 & 2.1 & \\
\hline & 3.97 & 0.0 & -2 . \\
\hline & 2.177761 & 0.00 & -2.7 \\
\hline & 0.00 & -3.9 & \\
\hline & -2.0 & & \\
\hline & 3.27 & 2.07 & -1. \\
\hline & 0.00 & 3.91 & -0.9 \\
\hline & 3.272518 & -2.071759 & -1.010546 \\
\hline & -3.9 & & \\
\hline & 2.0 & 3.27 & \\
\hline & 0.00 & -4.97 & 0.5 \\
\hline & 0.000000 & -2.177761 & 2.755068 \\
\hline & 2.071759 & -3.272518 & 1.01 \\
\hline & & 2.0 & -1.010 \\
\hline & & -2.0 & \\
\hline & 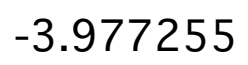 & 0.000000 & -2.62 \\
\hline
\end{tabular}

$H F=-928.7228197$ hartree 
Cartesian coordinate of optimized structure of $\mathbf{2}^{+}\left(D_{4 \mathrm{~h}}\right)$ at the B3LYP/6-31G(d) level

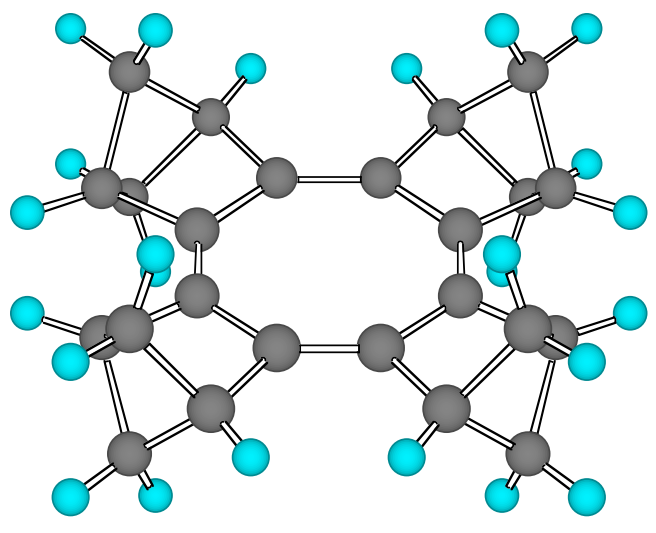
C $\quad 0.684306$
1.722847
0.000000
C 1.722847
0.684306
0.000000
C $1.722847-0.684306$
0.000000
C $0.684306-1.722847$
0.000000
C $-0.684306-1.722847$
0.000000
C $-1.722847-0.684306$
0.000000
C $-1.722847 \quad 0.684306$
0.000000
C $-0.684306 \quad 1.722847$
0.000000
C 1.534670
2.985913
0.000000
C 2.985913
1.534670
0.000000
C $2.985913-1.534670 \quad 0.000000$
C $1.534670-2.985913$
0.000000
C $-1.534670-2.985913$
0.000000
C $-2.985913-1.534670$
0.000000
C $-2.985913 \quad 1.534670$
0.000000
C -1.534670
2.985913
0.000000
C $2.615457 \quad 2.615457$
1.065496
C $2.6154572 .615457 \quad-1.065496$
C $2.615457-2.615457 \quad 1.065496$
C $2.615457-2.615457-1.065496$
$\begin{array}{llll}\text { C } & -2.615457 & -2.615457 & 1.065496\end{array}$
C $-2.615457-2.615457-1.065496$
C $-2.615457 \quad 2.615457 \quad 1.065496$ 


$\begin{array}{lrrr}\mathrm{C} & -2.615457 & 2.615457 & -1.065496 \\ \mathrm{H} & 1.029850 & 3.948314 & 0.000000 \\ \mathrm{H} & 3.948314 & 1.029850 & 0.000000 \\ \mathrm{H} & 3.948314 & -1.029850 & 0.000000 \\ \mathrm{H} & 1.029850 & -3.948314 & 0.000000 \\ \mathrm{H} & -1.029850 & -3.948314 & 0.000000 \\ \mathrm{H} & -3.948314 & -1.029850 & 0.000000 \\ \mathrm{H} & -3.948314 & 1.029850 & 0.000000 \\ \mathrm{H} & -1.029850 & 3.948314 & 0.000000 \\ \mathrm{H} & 2.280729 & 2.280729 & 2.051613 \\ \mathrm{H} & 3.385495 & 3.385495 & 1.159743 \\ \mathrm{H} & 2.280729 & 2.280729 & -2.051613 \\ \mathrm{H} & 3.385495 & 3.385495 & -1.159743 \\ \mathrm{H} & 2.280729 & -2.280729 & 2.051613 \\ \mathrm{H} & 3.385495 & -3.385495 & 1.159743 \\ \mathrm{H} & 2.280729 & -2.280729 & -2.051613 \\ \mathrm{H} & 3.385495 & -3.385495 & -1.159743 \\ \mathrm{H} & -2.280729 & -2.280729 & 2.051613 \\ \mathrm{H} & -3.385495 & -3.385495 & 1.159743 \\ \mathrm{H} & -2.280729 & -2.280729 & -2.051613 \\ \mathrm{H} & -3.385495 & -3.385495 & -1.159743 \\ \mathrm{H} & -2.280729 & 2.280729 & 2.051613 \\ \mathrm{H} & -3.385495 & 3.385495 & 1.159743 \\ \mathrm{H} & -2.280729 & 2.280729 & -2.051613 \\ \mathrm{H} & -3.385495 & 3.385495 & -1.159743 \\ & & \\ \mathrm{HF}=-928.5599615 \text { hartree } & \\ & & & \end{array}$

